\title{
Methodology of application of programmed training in the system of professional training of drivers
}

\author{
Evgeny Ageev ${ }^{1}$, Evgeny Vinogradov ${ }^{1, *}$, and Alexandr Novikov ${ }^{2}$ \\ ${ }^{1}$ South-West State University, 305040, 50 years of October str., 94, Kursk, Russia \\ ${ }^{2}$ Orel State University, 302026, Orel, Moscovskaya str., 77, Russia
}

\begin{abstract}
This article discusses an improved system for training candidates for drivers of vehicles, identifies factors that affect road safety in the conditions of the road network. The author's version of the development of a program of programmed training in the system of training candidates for drivers, which is focused on the ability to manage the process of mastering theoretical and practical material. The presented method makes it possible to increase the reliability of vehicle drivers, taking into account individual personal qualities, when performing exercises of road training, the experience of using programmed training in preparation for driving in real time is reflected.
\end{abstract}

\section{Introduction}

The annual growth in car production is characterized by high traffic intensity on roads and localities. At the same time, improving the design of cars, expanding the road network, and improving the quality of highways increases speed limits, complicates maneuvers, and increases the requirements for accuracy and speed of actions in road situations. All this requires drivers to have the knowledge and skill to control the vehicle in order to prevent the possibility of an emergency [1]. Analysis of the causes of road accidents allows us to identify the relationship of factors [2] contributing to the occurrence of such phenomena figure 1 .

The main factors that affect the high accident rate are: the driver's inability to analyze the traffic situation, which does not allow assessing the situation, making and implementing the right decision focused on traffic safety [3-5]. If the driver has analyzed all the necessary information, the violation of traffic rules may be caused by an incorrect assessment of the situation.

The inability to assess the situation is observed in drivers who did not receive the necessary training during the training process and were allowed to drive a vehicle. Failure to make the right decision may result from an unwillingness to drive a vehicle in such a way as to ensure the safety of others. The inability to implement the decision is the result of a lack of practical driving skills.

\footnotetext{
*Corresponding author: ganek09@rambler.ru
} 


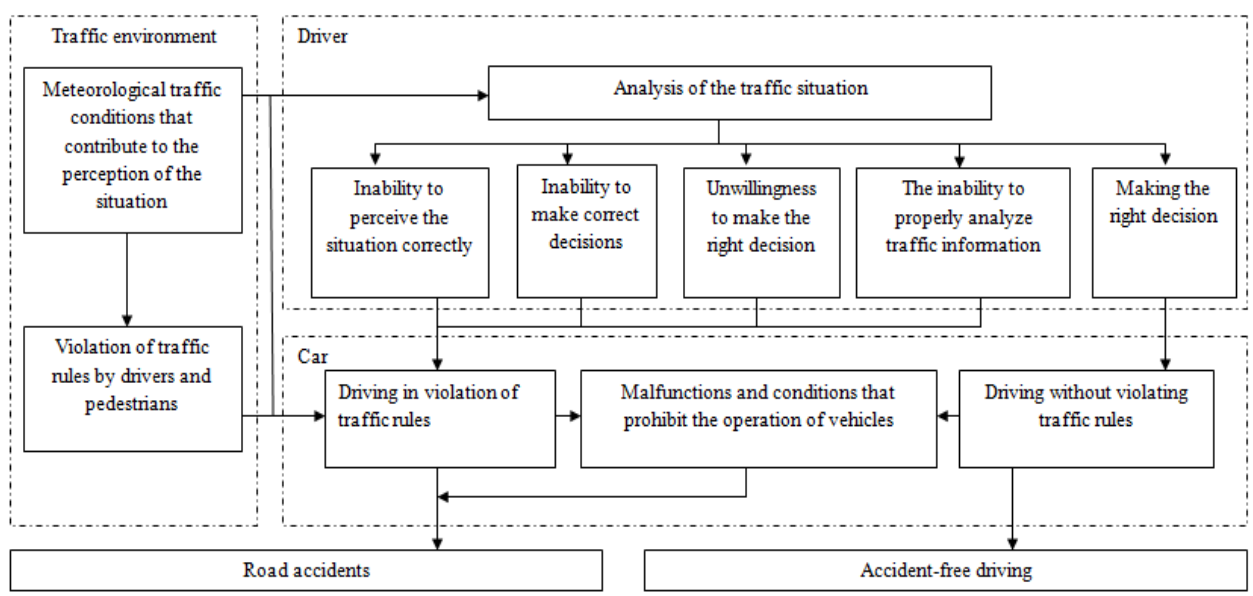

Fig. 1. Mechanism of occurrence of a traffic accident.

An emergency situation that is not related to the driver's actions may occur if there is interference on the road that does not have enough time to respond safely. Such interference is caused by drivers of other vehicles who violate traffic rules. Driving a technically defective vehicle in accordance with the list of faults and conditions that prohibit the operation of vehicles.

Analysis of statistical data on road accidents shows that most accidents occur for reasons related to the influence of the human factor, especially among novice drivers $[6,7]$.

At the same time, an important component is organized and purposeful work that is carried out in the system of professional training of drivers, focused on the experience of perception of the facts of the surrounding reality and safe behavior on the road.

In this regard, work is underway [8-10] on improving the effectiveness of training candidates for drivers focused on the principles of scientific analysis and management methods developed in Cybernetics, which allows us to consider the theory of training in a new way using the latest scientific data and technical tools [11].

Traditional methods of training do not allow us to meet the increasing requirements of the intensification of the educational process. The main disadvantage of traditional methods is the complexity of setting educational tasks, monitoring the degree of assimilation of the material, because they are not systematic, objective and stimulating. This disadvantage can be compensated by the introduction of programmed methods of training with use of technology [12-15].

The method of programmed training is a program for managing the process of mastering theoretical and practical skills formed by candidates for drivers, a rational sequence of the material being studied is determined and provides tasks and control questions that allow you to activate the learning process and monitor the quality of training.

In the theory and practice of programmed learning, there are three types of training programs: linear, branched, and combined (mixed). Linear training programs focus on the capabilities of weak candidates for drivers, while the training material is the same for everyone without taking into account individual abilities, so by the middle of the course, strong students stop attending classes without having mastered the course program in full. With extensive programming of educational material, a high degree of individualization of training in General is achieved, but also more economic training of groups, and achievement of the established level of training is guaranteed. Combined programs include linear and branched types of the programmed learning material at the same time. 
With a well-designed program, you can train large groups in the theoretical and practical sections of the driver candidate training program using machine and machine-free programming of the training material.

Machine programmed learning is based on the use of various technical devices of PC systems, tablets with installed software. Machine-based programmed learning uses training manuals, programmed textbooks, route maps, and control maps. At the same time, the effectiveness of programmed training depends on the quality of the training program and the way it is implemented.

The aim of the work is to improve the system of training vehicle operators through the use of programmed training.

\section{Materials and techniques}

The study performed using the method of programmed training allows monitoring the performance of candidates for drivers of basic operator actions related to the psycho physiological foundations of work, which are focused on their future professional activities and determining the main functions of the body that affect road safety.

Let's look at the experience of programmed driving training in a closed area on the example of working out simple driving skills when driving a car.

The development of the program of experimental programmed training was carried out in the following stages figure 2 .

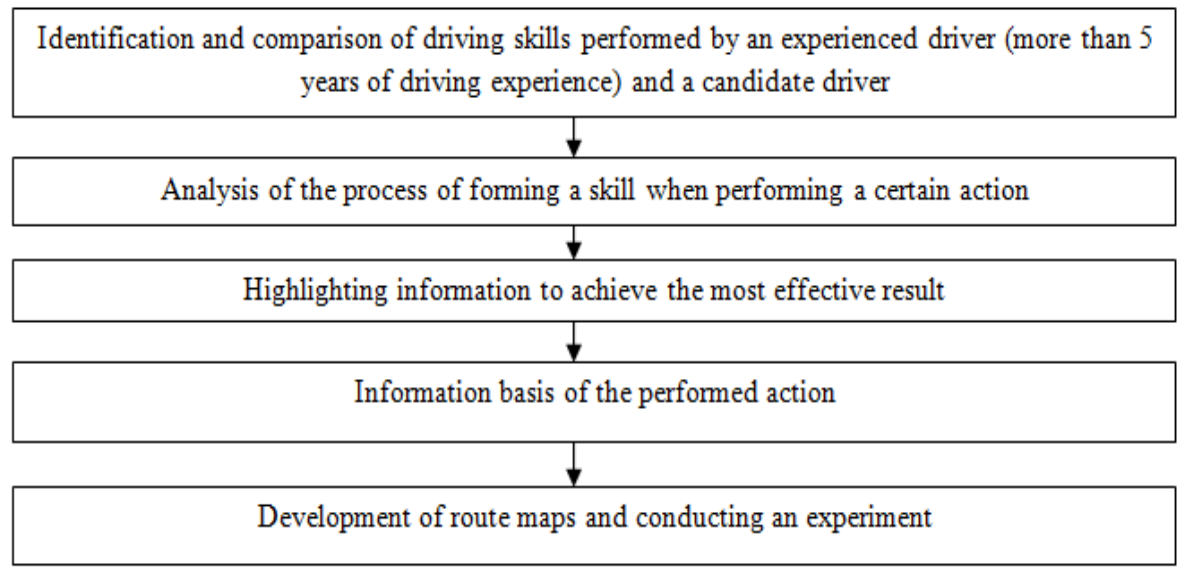

Fig. 2. Stages of developing an experimental programmed training program.

Based on the analysis of actions during the initial driving of the vehicle, as well as factors that affect this movement, the following is established:

1. The Ability to withstand straight traffic is directly dependent on the driver's ability to evaluate:

- the section of road that is used for traffic;

- spatial and geometric diagram of the car;

- position of the car on the road;

- moving the steering wheel depending on the vehicle's position estimates while driving.

2. The Assessment of these characteristics, the parameters of the car, the road and the position of the vehicle at each moment of movement is determined depending on the presence of signs by which the driver evaluates all components. 
3. The ability of the driver to operate the steering wheel in a straight line on the road depends on the following factors:

- the ability to make turns of the steering wheel in accordance with the choice of speed, dynamics of movement of the center of gravity, depending on the longitudinal axis of symmetry relative to the direction of movement;

- the ability of the driver to perform proactive steering wheel turns depending on the road situation;

- the success of the actions performed depends on the norms of rolling the clock when driving a vehicle, the design and adjustment of the steering system, the dynamics of the car's behavior, and other factors.

4. A comparative analysis of activities in a straight line direction of movement allows a candidate driver to identify the crucial dependence of maintaining the required course, depending on the information base of the action when managing the car-driving environment system.

5. When analyzing the interaction with the steering wheel, candidates for drivers have established the dependence of the actions performed and is determined by the amount of attention loading, using natural information, the operator is able to perform the main functions associated with driving, when attention is focused on a large number of different objects, the exercise is performed with considerable difficulty.

These facts, taking into account the content of the information revealed by current and novice drivers in straight-line traffic, formed the basis for the development of route maps and the process of experimental training table 1.

Table 1. Criteria for professional readiness of candidates for drivers.

Interaction object: car-driving
environment.
Actions performed: assessment of the
position and movement of the car on
the road, correction of actions
performed using the steering wheel.
$\begin{aligned} & \text { Task: training to maintain a straight } \\ & \text { direction of movement of the car with } \\ & \text { the help of auxiliary landmarks. } \\ & \text { The sequence of actions performed: the } \\ & \text { master of industrial training gives } \\ & \text { candidates for drivers a programmed } \\ & \text { manual with route training maps and } \\ & \text { demonstrates techniques for } \\ & \text { maintaining the straight direction of } \\ & \text { movement of the car in accordance with } \\ & \text { the training map, indicating the } \\ & \text { landmarks that you need to navigate } \\ & \text { when driving, taking into account the } \\ & \text { speed and dynamics of its displacement } \\ & \text { relative to the set course of movement. } \\ & \text { Then the candidate driver gets } \\ & \text { acquainted with the contents of the } \\ & \text { route map and fulfills its instructions. }\end{aligned}$


Building a detailed diagram of the actions performed when driving the route map shows the tasks performed, taking into account the interaction objects. The route map is divided into several tasks (operations), which reflect a set of landmarks, and indicate the order of their use in the orientation, Executive, and control parts of the actions performed. To work with maps, they are performed on separate blocks in digital form and are indicated by a sequential number, which allows you to quickly find the necessary map in the program guide.

The given sequence of training and especially the principles of schematized orientation allow candidates for drivers to master the necessary skills much faster, which helps to adapt training for other exercises: making turns, driving in a limited space, learning maneuvers related to stopping at sidewalks, leaving the overpass, driving in limited areas, driving on narrow sections of roads.

The considered method of programmed driving training is based on the characteristics of driving actions identified in the course of the study, as well as training actions, and is the result of the implementation of the relevant principles of professional training of drivers.

\section{Results and discussions}

The study performed using a systematic approach allows us to determine the degree of formation of professional qualities of candidates for drivers in the process of training at the training center. Based on the use of the training rating, indicators that have an impact on road safety are formed.

In accordance with the proposed experimental method of training candidates for drivers, 20 people were surveyed, 10 of them (the experimental group) were trained using the method of programmed training in order to form professionally important skills necessary for drivers of vehicles, 10 people (the control group) were trained in the traditional form of training. Studies have shown that students who have received professional training in this technique are more adapted to driving a vehicle when performing control exercises on the racetrack compared to the control group figure 3 .

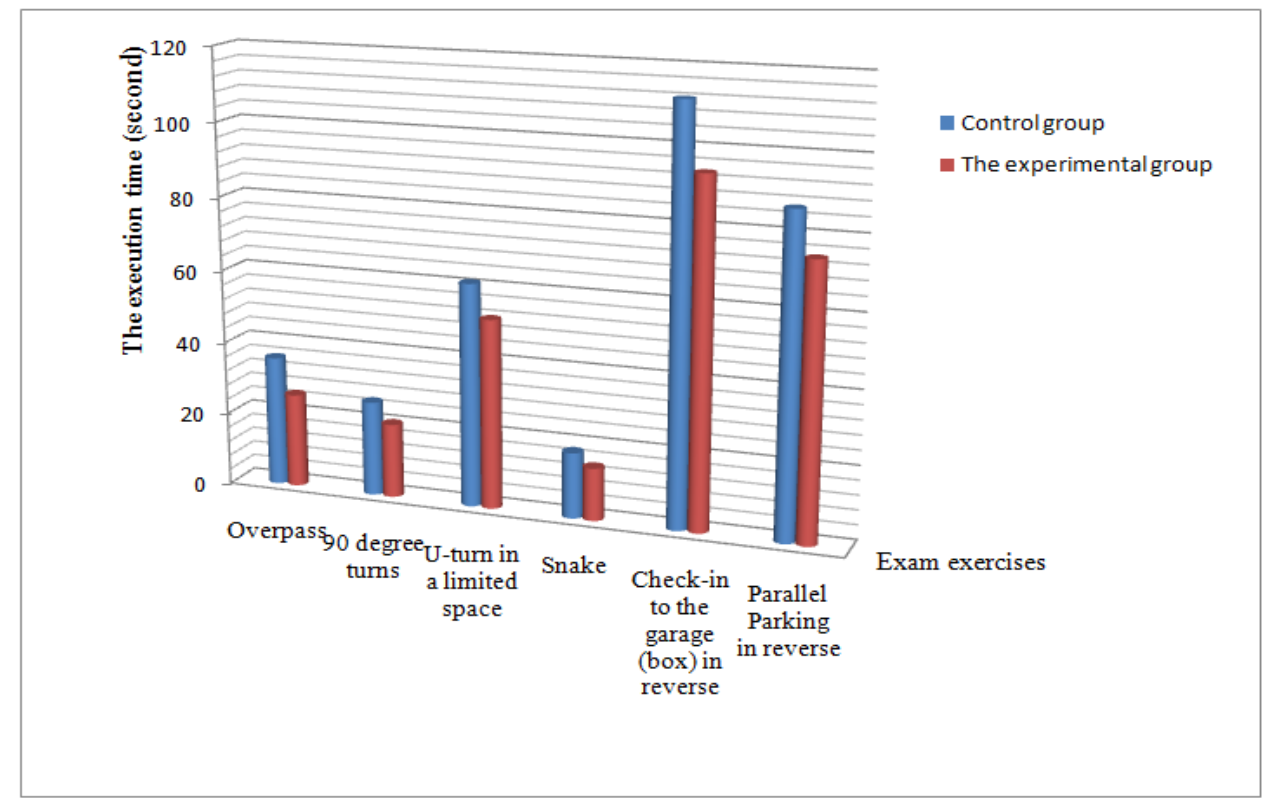

Fig. 3. Comparative diagram of the dynamics of performing exercises on the circuit. 
Professional training has many aspects and can be considered as an indicator of "professional skill", the purpose of which is to develop professional qualities that affect traffic safety before starting to drive a car independently.

Developed programmed route map exercises for the development of professional skills in the initial training stage at the training center, greatly complement the teaching materials program, and increase the reliability of diagnostic decision-making process.

One of the main principles of practical training is the principle of activity, which implies comprehensive development and deep awareness of their actions. In the system of professional training, there is an application of the method of exercises based on programmed training, comprehensive awareness of the process of learning motor skills and perception of reality.

To develop visual and motor skills when driving a car, you need to:

- inclusion in the training system of training elements of landmarks for the formation of special ocular assessments of the traffic environment, determining the position of the car in the traffic environment, performing preparatory work tasks when interacting with controls, combining visual and motor components into a complete driving skill.

- sequence of learning elements.

- use of technical means (artificial landmarks).

- schematization of landmarks and simplification of actions with them at all stages of formation of driving skills.

- exercise control of the exercises performed by the master of industrial training by mutual control on the part of the candidate driver and self-control.

As a result of the work done, practical skills related to driving a vehicle have significantly improved, and it has become possible to assess the degree of readiness for independent work, which has a positive effect in the field of road safety. The individual personal characteristics identified during the exercise should help to deepen the understanding of the role of the professional activity of the driver of the vehicle.

\section{Conclusion}

The use of improved methods of training candidates for drivers in the educational process gives positive results in reducing economic, environmental and social losses. The use of programmed training makes it possible to increase the level of professional fitness of drivers, which will undoubtedly affect road safety, and as a factor it reduces direct losses caused by accidents, creates safe driving conditions, increases labor productivity in the automotive industry, and increases the overall culture of behavior on the road.

\section{Acknowledgments}

The main task of professional training of drivers is the safety of professional activity on the roads. To date, driver training does not fully provide the perception of knowledge in the field of traffic regulations, requirements for safe driving, studying the device and design of the car, and there is also a low quality of practical training.

According to the results of the study and a comparative analysis of the training of candidates for drivers, the proposed method of programmed training allows you to master basic exercises much faster, and adaptation to the road network is carried out more comfortably. According to experts, the implementation of the program training methodology in the practice of professional training has reduced the number of mistakes made by $20 \%$ and reduced the time for performing control exercises in the conditions of road training. 


\section{References}

1 I. Pugachev, Y. Kulikov, G. Markelov, N. Sheshera, Analysis of Traffic Organization and Safety Systems, Transportation Research Procedia, v.20, pp. 529-535 (2017)

2 Global status report on road safety. Geneva: World Health Organization, 404 p. (2018)

3 V.F. Babkov, Road conditions and traffic safety, Transport, 271 p. (1993)

4 V. V. Lukyanov, Road safety, Transport, 262 p. (1983)

5 S. A. Evtyukov, Investigation and examination of road transport accidents, dnk $288 \mathrm{p}$. (2005)

6 E.V. Ageev, E.S. Vinogradov, Improving the system of training drivers of category "B", affecting road safety, World of transport and technological machines, v.4 (2019)

7 E.V. Ageev, E.S. Vinogradov, Principles and methods of evaluation of training complexes in the system of professional training of drivers, World of transport and technological machines, v.3 (2020)

8 A.N. Novikov, A.P. Tryashtsin, Y.N. Baranov, V.I. Samusenko, A.M. Nikitin, Evaluation of the effectiveness of the system of personnel training related to road safety Bulletin of the Bryansk state technical University, v.4, pp. 188-195 (2014)

9 D.X. French, Decision-making style, driving style, and self-reported involvement in road traffic accident Ergonomics, v. 36, pp. 627-644 (1993)

10 Y.I. Lobanova, Driving Style: determinants, characteristics, direction of optimization, Russian humanitarian journal, v.1, pp. 76-84 (2015)

11 A. Kaltenegger, New methods in driving instruction, CIECA Madrid (Spain) (2016)

12 H.A. Deery, Hazard and risk perception among young novice drivers, Journal of safety research, v.4, pp. 225-236. (2000)

13 M.G. Serikova, Improving the training of specialists for automobile transport enterprises, Transport business in Russia, v.3, pp.68-69 (2014)

14 A.V. Lyandenburskaya, I.S. Morozov, I.E. Ilina, V.V. Lyandenbursky, Requirements for digital modeling methods on a simulator, Ural scientific Bulletin, v.4, pp. 16-18 (2017)

15 A.Y. Yakimov, Improving the system of training drivers of motor vehicles on the basis of optimal definition of categories (types) of drivers (taking into account domestic and foreign experience), road Safety: collection of scientific papers of PKU SIC BJJ Ministry of internal Affairs of Russia, v.13, pp. 128-138 (2013) 